\title{
The Relationship between Adaptive Behavior and Cognitive Abilities (IQ) in Norwegian Children with Cerebral Palsy (CP): A Multi-Center Study
}

Gro Christine Christensen Løhaugen ${ }^{1 *}$, Cato Sundberg ${ }^{2}$, Harald Beneventi ${ }^{3}$, Ellen Elise Bakkan ${ }^{3}$, Guro L Andersen $^{4}$, Geir Walther ${ }^{5}$, Heidi Furre Østgård ${ }^{6}$, Torstein Vik ${ }^{6}$ and Jon Sverre Skranes ${ }^{6}$

${ }^{1}$ Department of Child Neurology and Rehabilitation, Sørlandet Hospital, Arendal, Norway

${ }^{2}$ Department of Pediatrics, Sørlandet Hospital, Arendal, Norway

${ }^{3}$ Department of Child Psychiatry, Stavanger University Hospital, Stavanger, Norway

${ }^{4}$ Department of Clinical and Molecular Medicine, Vestfold Hospital Trust, Tønsberg, Norway

${ }^{5}$ Department of Child and Adolescent Psychiatry, Vestfold Hospital Trust, Tønsberg, Norway

${ }^{6}$ Department of Clinical and Molecular Medicine, Norwegian University of Science and Technology, Trondheim, Norway

\begin{abstract}
Study background: To assess adaptive behavior and full IQ in children with Cerebral Palsy (CP) and explore cognitive and adaptive functioning within CP subtypes and associated problems, and in preterm born children.

Methods: Children with CP from seven Norwegian counties Norway registered in the Cerebral Palsy Register of Norway (CPRN) were invited. 65 children (mean age 10 years, 5 months (range 5-17), 41 males; 15\% had epilepsy and $39 \%$ were born preterm. The Vineland Adaptive Behavior scale 2nd edition (VABS) parental questionnaire and the Wechsler Intelligence Scale for Children $4^{\text {th }}$ edition were used.
\end{abstract}

Results: VABS scores and full IQ scores were lower than in Typically Developing (TD) children. Children with left-sided unilateral CP had higher VABS scores within the communication domain (mean:87, SD:13) than right-sided unilateral CP (mean:72, SD:13, $p=0.038$ ), but this was not significant when adjusted for IQ. More than $80 \%$ of the CP children obtained scores lower than expected for TD children within daily living skills. In the group with normal IQ (Full IQ $85-115, n=30$ ) $31 \%$ obtained scores as expected for age within daily life functioning.

Conclusion: Mean full IQ and level of adaptive functioning were lower in children with CP compared to TD children.

Keywords: Adaptive behaviour; IQ; Children with CP

\section{Introduction}

Cerebral Palsy (CP) represents one of the most common neurological conditions in childhood and is caused by abnormal brain development or injury of brain areas controlling movement and postural control. In 2007, the revised definition of CP presented by Rosenbaum et al. [1] included disturbances of sensation, perception, cognition, communication and behavior accompanying the motor problems, reflecting the complexity of the disorder. World-wide prevalence of $\mathrm{CP}$ has been estimated from 1.5 to 4 per 1000 live births [2]. A recent population-based multi-center study from Europe reported decreasing prevalence from 1.90 to 1.77 per 1000 live births from 1980 to 2003 [3]. In Norway, the prevalence was recently found to be 2.4 per 1000 live births [4].

Learning disorders and intellectual disability are more prevalent among children with CP than Typical Developing (TD) children [5]. The prevalence of cognitive impairment in children with CP, defined as a developmental (DQ) or Intelligence Quotient (IQ) below 70 was reported to be around $40 \%$ in a population based study from Iceland [6]. In a more recent study from Norway the prevalence of intellectual disability was $24 \%$ [7]. In the latter study intellectual disability was diagnosed in children with IQ scores below 70 who in addition had limitations of adaptive functioning, at least partly explaining the lower prevalence compared with other studies basing the diagnosis only on low DQ/IQ-scores. In addition to the motor and cognitive challenges, behavioral problems are found in as many as $48 \%$ of children with $\mathrm{CP}$ [8]. Less is known regarding adaptive functioning in everyday life in children with CP.

Adaptive behavior may be defined as how effective individuals meet the standard for personal independence and social responsibility that is expected for their age and cultural group [9]. This definition also implies that adaptive behavior is age appropriate, based on the expectation of others, typical functioning and not maximal performance, and is modifiable $[10,11]$ reported that social participation in children with mild CP, defined as being able to walk, and without learning disorders, did not deviate from peers [11]. However, epilepsy and speech disorders affected social participation negatively over time. We have in a previous study including 10 very preterm born children with CP and a mean IQ of 60 reported that adaptive behavior was closely related to cognitive function [12]. However, little is known regarding this relationship in the general population of children with CP.

On this background, the aim of the current study was to assess adaptive behavior in Norwegian children with CP by using the Vineland Adaptive Behavior Scale $2^{\text {nd }}$ edition (VABS) parental questionnaire and to explore any differences in adaptive functioning in children with $\mathrm{CP}$ born preterm or at term, with unilateral or bilateral CP, with or without epilepsy or with low versus normal IQ. We hypothesized that children with $\mathrm{CP}$ would be at a disadvantage compared TD children, especially regarding daily life activity that may be limited due to their reduced motor ability.

\section{Materials and Method}

The study is part of the intervention study "Working memory training

*Corresponding author: Gro Christine Christensen Løhaugen, Department of Child Neurology and Rehabilitation, Sørlandet Hospital, Arendal, Norway, Tel: +4737075750; E-mail: gro.lohaugen@sshf.no

Received July 12, 2018; Accepted August 07, 2018; Published August 14, 2018

Citation: Løhaugen GCC, Sundberg C, Beneventi H, Bakkan EE, Andersen GL, et al. (2018) The Relationship between Adaptive Behavior and Cognitive Abilities (IQ) in Norwegian Children with Cerebral Palsy (CP): A Multi-Center Study. Int J Neurorehabilitation 5: 328. doi: 10.4172/2376-0281.1000328

Copyright: (c) 2018 Løhaugen GCC, et al. This is an open-access article distributed under the terms of the Creative Commons Attribution License, which permits unrestricted use, distribution, and reproduction in any medium, provided the original author and source are credited. 
Citation: Løhaugen GCC, Sundberg C, Beneventi H, Bakkan EE, Andersen GL, et al. (2018) The Relationship between Adaptive Behavior and Cognitive Abilities (IQ) in Norwegian Children with Cerebral Palsy (CP): A Multi-Center Study. Int J Neurorehabilitation 5: 328 . doi: 10.4172/2376-0281.1000328

Page 2 of 6

in children with CP", ClinicalTrials.gov Identifier: NCT02119364 [13], a multicenter, randomized controlled trial of computerized working memory training (Cogmed) applying a stepped-wedge design [14]. The main outcome of the study was executive function, and learning abilities. As part of the general assessment of the participants, the Vineland Adaptive Behavior Scale (VABS) was administered to the parents. The results from the intervention study have not been published yet.

Children with CP born 1998-2009 living in seven counties in Norway and registered in the Cerebral Palsy Register of Norway (CPRN) were invited to participate. The Cerebral Palsy Register of Norway (CPRN) is an informed consent based national quality register established in 2006, and aims to record detailed information on all children with CP born in Norway since 1996. Information is reported at diagnosis, at 5 years and at 15-17 years of age [15]. Neuropediatric rehabilitation centers at hospitals in Norway provide summary and detailed data about the children. The reason for including only participants from seven counties was based on geographical considerations (travel distance), collaborative experience and neuropsychological competence. Since the intervention study required use of a computer, included children needed to be able to understand and use a game-like computer task, and those with the most severe motor impairments (Gross Motor Function Classification System level V) or with severe vision/hearing impairment or photosensitive epilepsy were excluded.

The $2^{\text {nd }}$ edition of the VABS parental questionnaire has been normed in Scandinavia for children from 2 years of age to 21 years and 11 months [10]. The parental questionnaire was administered to assess the child's daily life functioning within three main domains: communication, daily life and social skills, and these three domains together with the total score; General Adaptive Function (GAF) represents our main outcomes. Within each of the three domains there are three subscales (Figure 1). The communication domain contains questions regarding impressive and expressive language as well as reading and writing. The daily life domain focuses on the child's ability to function independently within the personal, domestic and community domain, and the social skills domain contains the subscales interpersonal relation, play and

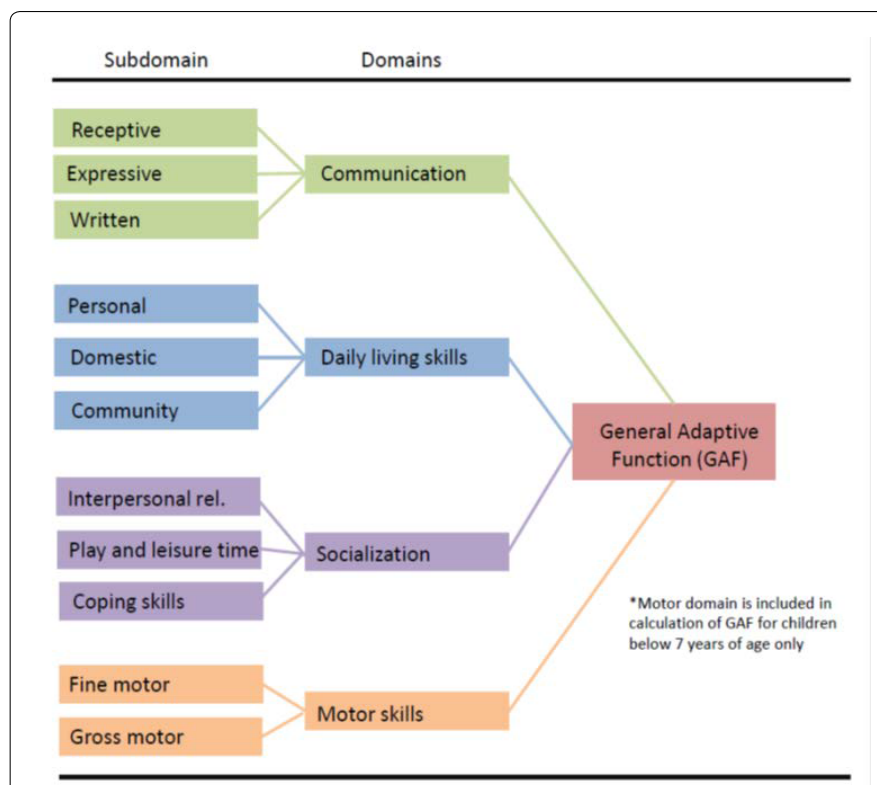

Figure 1: Internal structure of the Vineland Adaptive Behavior Scales $2^{\text {nd }}$ ed (VABS-II) leisure time and coping skills (Supplemental Table 1). For children up to 6 years, motor domain is included in calculating the total score "general adaptive function" (GAF) (Figure 1). The VABS also contains questions regarding maladaptive internalizing and externalizing behavior but this domain does not have Scandinavian norms and parents were not asked to complete this part of the questionnaire.

General cognitive ability (IQ) was assessed by the Wechsler Intelligence scale for children, $4^{\text {th }}$ edition (WISC-IV) [16]. Age appropriate Scandinavian norms were applied and all tests were performed by trained neuropsychologists. The core battery of the WISC-IV was applied including 10 subtests. For children with motor problems the block design subtest was interchanged for the picture completion task and the coding subtask was interchanged by the cancellation task, based on the individual child's abilities. The Wechsler Preschool and Primary Scale of Intelligence $-3^{\text {rd }}$ edition (WPPSI-III) [17] was applied for one child less than 6 years of age. Full IQ scores were included in the analysis. Normal IQ and VABS index/GAF scores are expected to be within +/- 1 Standard Deviation (SD) from mean (Mean Full IQ/VABS indices/GAF score=100); i.e. between 85 and 115. Low IQ/VABS indices/GAF scores are scores 70 to 84 . Scores less than 70 indicate cognitive impairment/adaptive malfunctioning are seen in children with intellectual disability. For the VABS subscales v-scores are calculated based on age. For typically developing children mean is 15 (SD:3). Scores between 12 and 18 are regarded as within expected for age, scores between 8 and 11 are low while scores below 8 indicate impaired adaptive functioning.

Socioeconomic status was assessed by Hollingshead Two Factor Index of Social Position that is based on the combined education and occupation status for both parents [18]. A short interview was performed with one of the parents for information about the child's birth weight, gestational age at birth and if the child had epilepsy.

Statistical analysis was performed with the Statistical Package for Social Sciences software, version 21 (IBM SPSS Statistics, Chicago, Illinois). Differences between group means were compared with the Student's t-test for normally distributed variables. For variables not normally distributed the Mann-Whitney $U$ test was applied. A univariate general linear model was used to compute mean values adjusted for Full IQ when comparing those with left and right unilateral $\mathrm{CP}$ with group as a fixed factor and Full IQ as covariate. Spearman's rho was used to evaluate the correlation between Full IQ and GAF.

The Regional Committee for Medical Research Ethics (Norwegian Health Region II) approved the study protocol (project number: 2012/298). Parents of the CP children that were invited to participate had previously consented to be registered and to receive information about relevant research studies through The Cerebral Palsy Register of Norway (CPRN). Written information both for the parents and the children, including consent forms was sent to the families by mail from the CPRN. The consent forms were returned to the CPRN to be transferred to the principal investigator of the research study.

\section{Result}

A total of 493 families were invited to participate in the study. Of these, 69 families (14\%) consented and 65 completed the VABS. The children's mean age when the assessment was completed was 10.3 (SD:3.0) years; 41 children (64\%) were boys and 25 (39\%) were born preterm. Ten children (15\%) had epilepsy. The dominating subtypes were spastic unilateral $(\mathrm{N}=34)$ and spastic bilateral $(\mathrm{N}=31)$ CP. Background data of all included children as well as for the two dominating CP subtypes are shown in Table 1, whereby information 
Citation: Løhaugen GCC, Sundberg C, Beneventi H, Bakkan EE, Andersen GL, et al. (2018) The Relationship between Adaptive Behavior and Cognitive Abilities (IQ) in Norwegian Children with Cerebral Palsy (CP): A Multi-Center Study. Int J Neurorehabilitation 5: 328 . doi: $10.4172 / 2376-0281.1000328$

Page 3 of 6

\begin{tabular}{|c|c|c|c|c|}
\hline Clinical characteristic & $\begin{array}{c}\text { CP } \\
\text { Total group } \\
n=65 \\
\text { Mean (SD) }\end{array}$ & $\begin{array}{c}C P \\
\text { Unilateral } \\
n=34 \\
\text { Mean (SD) }\end{array}$ & $\begin{array}{c}\text { CP } \\
\text { Bilateral } \\
n=31 \\
\text { Mean (SD) }\end{array}$ & $\begin{array}{c}\text { p-value } \\
\text { Unilateral } \\
\text { vs } \\
\text { Bilateral CP }\end{array}$ \\
\hline Mean (SD) birth weight (grams) & 2604 (996) & $2714(879)$ & $2487(1111)$ & 0.482 \\
\hline Mean (SD) gestational age (weeks) & $36(5)$ & $37(4)$ & $35(6)$ & 0.299 \\
\hline Preterm born n (\%) & $25(38.5)^{\star}$ & $10(29.4)$ & $15(48.4)$ & 0.203 \\
\hline Gender male n (\%) & $41(63.1)^{*}$ & $22(64.7)$ & $19(61.3)$ & 0.802 \\
\hline Socio-economic status $(\mathrm{n}=64)$ & $3.8(1.0)$ & $3.8(0.2)$ & $3.8(0.2)$ & 0.321 \\
\hline Age at assessment, years & $10.3(3.0)$ & $10.4(2.9)$ & $10.3(3.1)$ & 0.808 \\
\hline Epilepsy Yes n (\%) (n=64) & $10(15.4)$ & $5(14.7)$ & $5(16.1)$ & 1.000 \\
\hline
\end{tabular}

Mann-Whitney $U$ test for non-parametric variables, Chi-square test for categorical variables.

Abbreviations: SD, standard deviation; IQ, Intelligence Quotient

^ Missing data for 2 children due to adoption

aMissing data for 5 children

Table 1: Clinical characteristics in the total group of children with cerebral palsy $(C P)(n=65)$, and within subgroups with spastic unilateral $C P$

\begin{tabular}{|c|c|c|c|c|c|}
\hline $\begin{array}{l}\text { Index/subscale } \\
\text { Mean (SD) }\end{array}$ & Total $\mathrm{CPn}=65$ & Ref range TD & $\begin{array}{l}\text { USCP } \\
n=34\end{array}$ & $\begin{array}{c}\mathrm{BSCP} \\
\mathrm{n}=31\end{array}$ & $p$-value ** \\
\hline Communication index* & $78(17.3)$ & $85-115$ & $77(14)$ & $79(20)$ & 0.898 \\
\hline Receptive & $12.2(3.4)$ & $12-18$ & $12.1(3.3)$ & $12.4(3.7)$ & 0.787 \\
\hline Expressive & $11.9(2.8)$ & $12-18$ & $11.7(2.5)$ & $12.2(3.1)$ & 0.539 \\
\hline Reading and writing & $10.8(3.4)$ & $12-18$ & $10.7(3.1)$ & $10.8(3.8)$ & 0.824 \\
\hline Daily living skills index* & $74.9(14.3)$ & $85-115$ & $73(12)$ & $76(16)$ & 0.520 \\
\hline Personal & $9.4(3.2)$ & $12-18$ & $9.2(2.3)$ & $9.7(4.0)$ & 0.390 \\
\hline Domestic & $11.4(2.7)$ & $12-18$ & $11.4(2.3)$ & $11.4(3.2)$ & 0.852 \\
\hline Community & $10.8(2.8)$ & $12-18$ & $10.3(2.5)$ & $11.3(3.1)$ & 0.199 \\
\hline Socialization index* & $83.2(15.7)$ & $85-115$ & $80(13)$ & $87(17)$ & 0.106 \\
\hline Interpersonal relations & $12.2(3.5)$ & $12-18$ & $11.6(3.0)$ & $12.8(3.9)$ & 0.146 \\
\hline Play and leisure & $11.8(2.8)$ & $12-18$ & $11.4(2.3)$ & $12.2(3.2)$ & 0.352 \\
\hline Coping skills & $12.6(3.0)$ & $12-18$ & $11.9(2.3)$ & $13.3(3.4)$ & 0.152 \\
\hline General Adaptive Function* & $77.1(14.4)$ & $85-115$ & $76(9)$ & $78(18)$ & 0.427 \\
\hline
\end{tabular}

Mann Whitney U test for non-parametric variables. SD: Standard Deviation; CP: Cerebral Palsy.

${ }^{*}$ Standard scores **for BSCP versus USCP.

Table 2: Vineland Adaptive Behavior Scale-II results. Index and composite standard scores and subscale V-scores in the total group of children with cerebral palsy (CP), and among children with Unilateral Spastic (USCP) and Bilateral Spastic (BSCP) CP. For comparison the reference range (i.e. mean +/- 1SD) for Typical Developing (TD) children in Norway is included in the table. Mean and standard deviation for typically developing children: Index scores mean100, SD 15. Subscale scores: Mean 15, SD

3. Scores as expected for age range: $85-115$ for index scores, $12-18$ for subscale scores. Index scores below 70 and subscale scores below 8 would indicate impairment.

on birth weight and gestational age was missing for two children who had been adopted by their parents. The mean IQ of the 60 children who had a formal IQ test was 83 (SD:17). Of these, 30 (50\%) had normal IQ, while $32 \%$ had low IQ and $18 \%$ had IQ scores below 70 indicating intellectual disability. Five children without formal IQ testing were included in analysis comparing children with normal vs low IQ based on clinical assessment.

There was no difference in mean IQ between children with unilateral (IQ: 84; SD:17) and bilateral CP (IQ: 83; SD:17; p=0.599). There was a moderate, but statistically highly significant correlation (Portney \& Watkins, 2009) between full IQ and GAF in the total CP group $(\mathrm{r}=0.444, \mathrm{p}=0.001)$.

Table 2 shows the mean scores of the VABS in the total group as well as for the two dominating CP subtypes. There were no significant differences in any of the scores between children with unilateral and bilateral CP. The mean VABS index and subdomain scores in the total group of children with $\mathrm{CP}$ and within the two $\mathrm{CP}$ subtypes were below - 1 SD compared with TD children.

To further explore the relationship between general cognitive ability and adaptive behavior, two groups were defined: Those with normal IQ (IQ $\geq 85, \mathrm{n}=30$ ) and with low IQ (IQ $<85, \mathrm{n}=33$ ). There were no differences between these groups regarding birth weight, gestational age at birth, SES or age at assessment (Supplemental Table 2). There were more children with epilepsy $(n=9)$ and fewer with unilateral CP $(n=13)$ among those with low IQ (Supplemental Table 2). When comparing the VABS subscale profile in the two groups, those with low IQ were at a disadvantage on all subscales, the differences reaching statistical significance on expressive language, reading and writing, domestic, community, interpersonal and play and leisure subscales, while children with normal IQ obtained scores within the normal range within communication and social functioning (Supplemental Figure 1). Regardless of IQ-level the lowest scores were found within the daily living domain. The frequency of low scores in the total group of children is shown in Figure 2.

Among children with unilateral $\mathrm{CP}$, there were no significant differences between those with left $(\mathrm{n}=11)$ and right sided affection $(n=23)$ regarding age at assessment, birth weight, gestational age or socioeconomic status. Children with left sided hemiparesis had a mean full IQ score of 91 (SD:13) compared with 81 (SD:17) among those with right sided paresis $(\mathrm{p}=0.065)$, and on the VABS those with left sided affection had higher scores on the communication index (mean score left-sided: 87; SD:13, versus right-sided 72; SD:13; $\mathrm{p}=0.007$ ). All three subscales that constitute the communication index score; receptive, expressive and reading and writing were lower in those with right than left sided $\mathrm{CP}$, significantly so for the reading and writing subdomain 
Citation: Løhaugen GCC, Sundberg C, Beneventi H, Bakkan EE, Andersen GL, et al. (2018) The Relationship between Adaptive Behavior and Cognitive Abilities (IQ) in Norwegian Children with Cerebral Palsy (CP): A Multi-Center Study. Int J Neurorehabilitation 5: 328. doi: 10.4172/2376-0281.1000328

Page 4 of 6

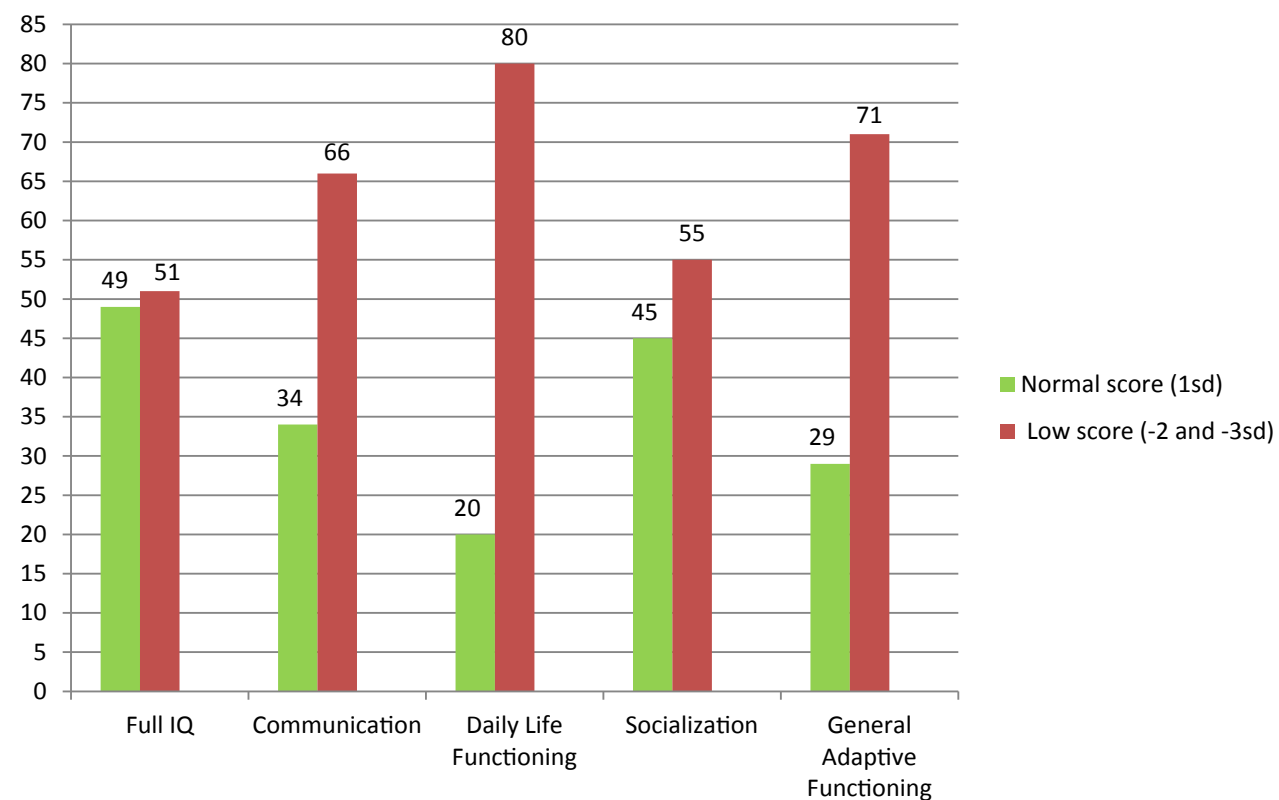

P.S: Green bars represent $\%$ of children obtaining scores within normal limits, red bars represent $\%$ of children with scores $-2 S D$ from expected mean.

Figure 2: Frequency (\%) of normal and low scores on Full IQ and VABS-II Indexes in the total group ( $n=65)$.

(mean 12, SD:3 in left unilateral vs. mean 10 SD:3 in right unilateral group; $\mathrm{p}=0.038$ ). When IQ was included as a covariate the differences between those with right and left sided $\mathrm{CP}$ in the communication index as well in the subscale reading and writing disappeared (data not shown).

Participants born preterm $(n=25)$ did not differ from those born at term $(n=38)$ in clinical characteristics apart from the expected difference in birth weight and gestational age (Supplemental material Table 3), and there were no significant differences regarding indices or subscale scores on the VABS (Supplemental material Table 4).

Children with epilepsy $(n=10)$ were older (mean age 12.6, SD:3.1) and obtained lower full IQ scores (mean: 66, SD:15) than those without epilepsy ( $n=54$; mean age:9.9; SD:2.8; $\mathrm{p}=0.03$; mean IQ: 87 ; SD:15 , $\mathrm{p}=0.001$ ), but there were no significant differences between these groups regarding any of the VABS indices or subscale scores (data not shown; information on epilepsy was missing in one child).

\section{Discussion and Conclusion}

We found that children with CP in the present study had adaptive functioning in the low range compared with TD peers, the lowest scores being obtained within the daily living skills domain. The level of adaptive functioning was largely in accordance with the cognitive abilities measured by full IQ, and adaptive functioning was similar in children with spastic unilateral and bilateral CP. Those with right unilateral CP were at a disadvantage compared to those with left unilateral $\mathrm{CP}$, the latter obtaining scores within normal range for typically developing children on full IQ, communication and social functioning.

Strength of the present study is the use of a validated questionnaire and a full, standardized IQ-test with reported norms for Norwegian children. There were no difference between groups regarding SES when comparing uni- and bilateral CP, preterm and term born and those with low IQ and normal IQ. However, due to our inclusion criteria those with the most severe motor deficits were not participating. All assessments were performed by trained clinical neuropsychologists. Limitations are the small sample size, the heterogeneous study group and missing data. These factors restrict the number of associated factors that may be compared between groups as well as our statistical power. In some of the subgroup analyses, for example when comparing children with and without epilepsy and children with left and right sided hemiplegia, the groups were small, and lack of statistical significance should therefore be interpreted with caution. This also applies for the only statistically significant finding; the difference in reading and writing skills between left and right sided hemiplegia. However, there was a trend towards lower scores in all domains and subdomains in those with right sided hemiplegia compared with left-sided. We excluded children unable to use a computer and to understand the task required for the intervention study. Thus, our results are probably not representative for all children with CP, but mainly for children with motor impairments corresponding to GMFCS levels I-IV. Moreover, only $14 \%$ of the invited families participated in the study. However, those who chose to participate committed themselves and their children to participate in a five week intensive training computer based program of working memory, and we therefore consider it most unlikely that we have studied a selected group of children with poor adaptive function. Finally, the proportion of children with intellectual disability was low in the present study, compared with the literature [19], and thus, our results are more likely to over- than to under-estimate adaptive functioning in children with CP.

The relationship between general cognitive ability and adaptive behavior is well known [10] but less explored in children with CP. For the total group, correlation between Full IQ and GAF scores were considerable. Still, about fifty percent obtained a low Full IQ score, while the prevalence of low GAF score were more than $70 \%$. This may reflect a discrepancy between cognitive ability and general adaptive function. This finding is in contrast to that of Fjørtoft et al. [12], reporting GAF scores higher than IQ. Their sample were smaller $(n=10)$ and included children with sensory deficits (reduced hearing) and one child with 
Citation: Løhaugen GCC, Sundberg C, Beneventi H, Bakkan EE, Andersen GL, et al. (2018) The Relationship between Adaptive Behavior and Cognitive Abilities (IQ) in Norwegian Children with Cerebral Palsy (CP): A Multi-Center Study. Int J Neurorehabilitation 5: 328. doi: 10.4172/2376-0281.1000328

Page 5 of 6

GMFCS level V. Mean Full IQ was also lower $(\mathrm{IQ}=60)$ than in our sample and all children were born preterm that in itself represents a risk of cognitive deficits.

More than $80 \%$ of the total CP group obtained a score below what is expected for typically developing children on the daily life skills domain and this domain was the one that deviated most from expected mean. Daily living skills assessed with the VABS include questions regarding eating, dressing, maintaining personal hygiene, household tasks, understanding time and money, and the use of computers. The motor impairments of children with CP may explain the lower result within this domain. In a larger sample of adolescents with CP $(n=103)$ comparable to our population, except that their population was slightly older (16-20 years). Donkerwoort et al. [20], found that only $46 \%$ were restricted in daily life skills assessed by VABS, compared to $80 \%$ in our group. The VABS scores in that study reflecting better daily life functioning may be explained by the difference in age (16-20 years) with more opportunity and expectations regarding independence in their population than in our younger population.

We also divided our group into those with normal and low IQ to explore the VABS profile. About half of the participants had low IQ, and as expected this subgroup also obtained lower scores on the VABS indicating less independence and more need for practical support regarding all aspects of adaptive functioning; communication, social and daily life functioning. This is in accordance with a follow-up study of more than 400 Dutch children and adolescents with CP reporting that intellectual level, GMFCS level, additional health issues (sensory problems, epilepsy) contributed to level of independence in daily living activities [21] and social participation [11]. Vos et al. (2014), reported that expressive communication was closely related to type of motor disorder, while receptive communication was closely related to intellectual disability [22].

We found that $32 \%$ of our participants obtained IQ scores that may be related to more specific learning disorders based on low IQ (i.e. between 85 and 70). In clinical terms this subgroup may be in need of intervention in school and some level of support in daily life.

Children with left unilateral CP obtained higher VABS scores on reading and writing, resulting in improved communication index scores compared to those with right unilateral CP. This is most likely a secondary effect from those who are affected on the right side of the body having to use their left hand as their primary writing hand, resulting in pathological left handedness and poorer writing function than for those that could use their dominant hand freely. In addition those with right sided hemiplegia scored lower on Full IQ by 10 points. When adjusting for the 10 point Full IQ difference, the differences in VABS scores were not significant.

About $40 \%$ of our study group was born preterm, and preterm birth represents increased risk of adverse cognitive [23] and functional outcome [24]. However, in our study group there were no significant differences between preterm and term born children on cognitive and VABS measures. We did not find lower VABS scores in those with epilepsy, but given the very small sample of 10 children with epilepsy negative results must be interpreted with caution.

Our findings imply that children with $\mathrm{CP}$ have adaptive functioning in accordance with their general cognitive ability. However, most children with CP had challenges regarding daily living skills. To be able to describe and tailor the correct interventions to the individual child with $\mathrm{CP}$, performing both cognitive and adaptive assessment seems expedient. Children with right unilateral CP obtained lower (not significant) scores across all indexes and on the GAF and also had lower Full IQ. This may indicate that addressing cognitive and adaptive functioning are especially important among this subgroup of children with right sided CP. Due to our inclusion criteria children with sensory deficits and/or GMFCS level V were not included.

\section{Acknowledgement}

This project has been made possible by the Norwegian Extra Foundation for Health and Rehabilitation. We thank all the children and their families for participating in this study, the Cerebral Palsy Register of Norway (CPRN) and the Norwegian Cerebral Palsy Association for their help, support and interest in this project. We thank Clinical Neuropsychologists Kristin Bostrøm and Truls Fagernes Olsen at the Child Habilitation Center, Vestfold Hospital Trust, Tønsberg, Norway for participating in the cognitive and adaptive assessments in Vestfold. We also thank Secretary Siv Stigen and researcher Knut Jørgen Bjuland at the department of Pediatrics, Sørlandet Hospital, Arendal for support during the submission process of this paper.

\section{References}

1. Rosenbaum P, Paneth N, Leviton A, Goldstein M, Bax M, et al. (2007) A report The definition and classification of cerebral palsy April 2006. Dev Med Child Neurol Suppl 109: 8-14.

2. Arneson CL, Durkin MS, Benedict RE, Kirby RS, Yeargin-Allsopp M, et al (2009) Prevalence of cerebral palsy: Autism and Developmental Disabilities Monitoring Network, three sites, United States, 2004. Disabil Health J 2: 45-48.

3. Sellier E, Platt MJ, Andersen GL, Krageloh-Mann I, De La Cruz J, et al. (2016) Decreasing prevalence in cerebral palsy: A multi-site European populationbased study, 1980 to 2003. Dev Med Child Neurol 58: 85-92.

4. Hollung SJ, Vik T, Wiik R, Bakken IJ, Andersen GL (2017) Completeness and correctness of cerebral palsy diagnoses in two health registers: Implications for estimating prevalence. Dev Med Child Neurol 59: 402-406.

5. Jenks KM, de Moor J, van Lieshout EC (2009) Arithmetic difficulties in children with cerebral palsy are related to executive function and working memory. $J$ Child Psychol Psychiatry 50: 824-833.

6. Sigurdardottir S, Eiriksdottir A, Gunnarsdottir E, Meintema M, Arnadottir U, et al. (2008) Cognitive profile in young Icelandic children with cerebral palsy. Dev Med Child Neurol 50: 357-362.

7. Stadskleiv K, Jahnsen R, Andersen GL, von Tetzchner S (2018) Neuropsychological profiles of children with cerebral palsy. Dev Neurorehabil 2: $108-120$.

8. Sigurdardottir S, Indredavik MS, Eiriksdottir A, Einarsdottir K, Gudmundsson $\mathrm{HS}$, et al. (2010) Behavioural and emotional symptoms of preschool children with cerebral palsy: A population-based study. Dev Med Child Neurol 52: 1056 1061.

9. National Research Council (US) Committee on Disability Determination for Mental Retardation; Reschly DJ, Myers TG, Hartel CR, editors (2002) Mental retardation: Determining eligibility for social security benefits. Washington (DC): National Academies Press, US

10. Sparrow S, Chicchetti V, Balla A. Vineland-II (2005) Vineland Adaptive Behavior Scales, Second Edition.

11. Tan SS, van der Slot WM, Ketelaar M, Becher JG, Dallmeijer AJ, et al. (2016) Factors contributing to the longitudinal development of social participation in individuals with cerebral palsy. Res Dev Disabil 57:125-135.

12. Fjørtoft T, Grunewaldt KH, Løhaugen GC, Morkved S, Skranes J, et al. (2015) Adaptive behavior in 10-11 year old children born preterm with a very low birth weight (VLBW). Eur J Paediatr Neurol 19: 162-169.

13. Løhaugen GC, Beneventi H, Andersen GL, Sundberg C, Ostgard HF, et al. (2014) Do children with cerebral palsy benefit from computerized working memory training? Study protocol for a randomized controlled trial. Trials 15: 269.

14. Brown CA, Lilford RJ (2006) The stepped wedge trial design: A systematic review. BMC Med Res Methodol 6: 54.

15. Andersen GL, Irgens LM, Haagaas I, Skranes JS, Meberg AE, et al. (2008) Cerebral palsy in Norway: Prevalence, subtypes and severity. Eur J Paediatr Neurol 12: 4-13.

16. Wechsler D (2009) WISC-IV. Wechsler Intelligence Scale for children-4 $4^{\text {th }}$ ed Norwegian version: NCS Pearson, Inc. 
Citation: Løhaugen GCC, Sundberg C, Beneventi H, Bakkan EE, Andersen GL, et al. (2018) The Relationship between Adaptive Behavior and Cognitive Abilities (IQ) in Norwegian Children with Cerebral Palsy (CP): A Multi-Center Study. Int J Neurorehabilitation 5: 328 . doi: $10.4172 / 2376-0281.1000328$

Page 6 of 6

17. Wechsler D (2008) WPPSI-III. Wechsler preschool and primary scale of intelligence- $3^{\text {rd }}$ ed. Norwegian version: NCS Pearson, Inc.

18. Hollingshead AB (1957) Two factor index of social position. Mimeo, New Haven, Connecticut: Yale University.

19. Bottcher L, Flachs EM, Uldall P (2010) Attentional and executive impairments in children with spastic cerebral palsy. Dev Med Child Neurol 52: e42-e47.

20. Donkervoort M, Roebroeck M, Wiegerink D, van der Heijden-Maessen $\mathrm{H}$, Stam $H$, et al. (2007) Determinants of functioning of adolescents and young adults with cerebral palsy. Disabil Rehabil 29: 453-463.

21. Vos RC, Becher JG, Ketelaar M, Smits DW, Voorman JM, et al. (2013)
Developmental trajectories of daily activities in children and adolescents with cerebral palsy. Pediatrics132: e915-e923.

22. Vos RC, Dallmeijer AJ, Verhoef M, Van Schie PE, Voorman JM, et al. (2014) Developmental trajectories of receptive and expressive communication in children and young adults with cerebral palsy. Dev Med Child Neurol 56: 951 959.

23. Løhaugen GCC, Gramstad A, Evensen KAI, Martinussen M, Lindqvist S, et al (2010) Cognitive profile in young adults born preterm at very low birthweight. Dev Med Child Neurol 52: 1133-1138.

24. Hielkema T, Hadders-Algra M (2016) Motor and cognitive outcome after specific early lesions of the brain: A systematic review. Dev Med Child Neurol 58: 46-52. 